\title{
Long-time behaviour of non-local in time Fokker-Planck equations via the entropy method
}

\author{
Jukka Kemppainen* and Rico Zacher ${ }^{\dagger}$
}

\begin{abstract}
We consider a rather general class of non-local in time Fokker-Planck equations and show by means of the entropy method that as $t \rightarrow \infty$ the solution converges in $L^{1}$ to the unique steady state. Important special cases are the time-fractional and ultraslow diffusion case. We also prove estimates for the rate of decay. In contrast to the classical (local) case, where the usual time derivative appears in the Fokker-Planck equation, the obtained decay rate depends on the entropy, which is related to the integrability of the initial datum. It seems that higher integrability of the initial datum leads to better decay rates and that the optimal decay rate is reached, as we show, when the initial datum belongs to a certain weighted $L^{2}$ space. We also show how our estimates can be adapted to the discrete-time case thereby improving known decay rates from the literature.
\end{abstract}

AMS subject classification: 35R11, 45K05, 47G20

Keywords: temporal decay estimates, non-local Fokker-Planck equation, entropy method, fractional derivative, subdiffusion, time-discrete scheme

\section{Introduction and main result}

We study the large time behaviour of solutions to the non-local in time Fokker-Planck equation

$$
\partial_{t}\left(k *\left[u-u_{0}\right]\right)-\nabla \cdot(\nabla u+u \nabla V)=0, \quad t>0, x \in \mathbb{R}^{d},
$$

where $u=u(t, x)$,

$$
\left.u\right|_{t=0}=u_{0}, x \in \mathbb{R}^{d},
$$

is the initial datum, $V=V(x)$ is the given potential and $k * v$ denotes the convolution on the positive halfline $\mathbb{R}_{+}:=[0, \infty)$ w.r.t. the time variable, that is $(k * v)(t)=\int_{0}^{t} k(t-\tau) v(\tau) d \tau$, $t \geq 0$.

The kernel $k \in L_{\text {loc }}^{1}\left(\mathbb{R}_{+}\right)$belongs to a certain class of kernels (it is of type $\mathcal{P C}$, see Section 2.1 ), which covers most of the relevant integro-differential operators w.r.t. time that appear in physics in the context of so-called subdiffusion processes, in particular the time-fractional case, where

$$
k(t)=\frac{t^{-\alpha}}{\Gamma(1-\alpha)}, \quad t>0,
$$

and $\partial_{t}(k * v)$ coincides with the Riemann-Liouville fractional derivative $\partial_{t}^{\alpha} v$ of order $\alpha \in(0,1)$, see Section 2.1 below. Note that $\partial_{t}(k *[v-v(0)])=k * \partial_{t} v$ for sufficiently smooth functions $v$.

\footnotetext{
*J.K. was supported by the Vilho, Yrjö and Kalle Väisälä Foundation of the Finnish Academy of Science and Letters

$\dagger^{\dagger}$ R.Z. was supported by a research grant of the German Research Foundation (DFG), GZ Za 547/4-1.
} 
Fokker-Planck equations, also known as Kolmogorov forward equations, are a central subject in the theory of diffusion processes. They describe how the probability density function of the velocity (or some other observable like position) of a particle evolves with time. The non-local in time version (1) is used in physics for the modeling of subdiffusion processes. Here the mean square displacement $m(t)$ of a diffusive particle, which is defined by

$$
m(t)=\int_{\mathbb{R}^{d}}|x|^{2} Z(t, x) \mathrm{d} x, \quad t>0,
$$

with $Z(t, x)$ denoting the fundamental solution of equation (1) with $V=0$ and $\left.Z\right|_{t=0}=\delta_{0}$ (see [10]), grows slower as $t \rightarrow \infty$ than in the classical case of Brownian motion, where $m(t)=c t$, $t>0$, with some constant $c>0$. For example, in the time-fractional diffusion case $m(t)=c t^{\alpha}$ (cf. [17]). Another example, which is also covered by our setting, is ultraslow diffusion (see Example 4.3 below); here $m(t)$ merely grows logarithmically for large times.

In the time-fractional case, equation (1) may be viewed as the master equation for the probability density of the scaling limit of a random walk with a heavy-tailed waiting time distribution or as an overdamped limit of a fractional Klein-Kramers equation. For details we refer to [17, 18]. Concerning the stochastic viewpoint of (1) in this case, we first recall that the stochastic differential equation (SDE) corresponding to the classical Fokker-Planck equation

$$
\partial_{t} u-\nabla \cdot(\nabla u+u \nabla V)=0, \quad t>0, x \in \mathbb{R}^{d},
$$

is given by

$$
\mathrm{d} X_{t}=-\nabla V\left(X_{t}\right) \mathrm{d} t+\sqrt{2} \mathrm{~d} B_{t}
$$

where $B_{t}$ denotes Brownian motion. Let $D_{t}$ be an $\alpha$-stable scaling limit of the waiting time process and let

$$
E_{t}=\inf \left\{\tau>0: D_{\tau}>t\right\}
$$

be its inverse or the first hitting time process. Then the time-changed process $Y_{t}=X_{E_{t}}$ satisfies the SDE

$$
\mathrm{d} Y_{t}=-\nabla V\left(Y_{t}\right) \mathrm{d} E_{t}+\sqrt{2} \mathrm{~d} B_{E_{t}}
$$

and the transition probability density $u$ is the solution of (1) with $k$ as in $(3)$, see $[7,16]$ for more details.

The slowness of subdiffusion processes for large times is reflected by lower temporal decay rates as well. These have been studied extensively in $[10,11]$ for solutions of (1) with $V \equiv 0$. For example, considering the time-fractional case (that is $k$ is given by $(3)$ with $\alpha \in(0,1)$ ) and assuming that $u_{0} \in L^{1}\left(\mathbb{R}^{d}\right) \cap L^{2}\left(\mathbb{R}^{d}\right)$ the solution $u$ of (1) with vanishing potential given by

$$
u(t, x)=\int_{\mathbb{R}^{d}} Z(t, x-y) u_{0}(y) \mathrm{d} y
$$

satisfies the (optimal) estimates

$$
\|u(t, \cdot)\|_{L^{2}\left(\mathbb{R}^{d}\right)} \lesssim t^{-\min \left\{\frac{\alpha d}{4}, \alpha\right\}}, \quad t>0, d \in \mathbb{N} \backslash\{4\}
$$

and

$$
\|u(t, \cdot)\|_{L^{2, \infty}\left(\mathbb{R}^{d}\right)} \lesssim t^{-\alpha}, \quad t>0, d=4
$$


where $L^{2, \infty}\left(\mathbb{R}^{d}\right)$ refers to the weak $L^{2}$ space, see [10]. This decay behaviour is markedly different from that in the case of the classical heat equation, where under the same assumptions $\|u(t, \cdot)\|_{L^{2}\left(\mathbb{R}^{d}\right)} \lesssim t^{-d / 4}, t>0$, in all dimensions $d \in \mathbb{N}$.

If the potential $V$ in (1) does not vanish and $e^{-V} \in L^{1}\left(\mathbb{R}^{d}\right)$ (which will be assumed throughout this paper), there is a unique steady state of (1) with unit mass, which is given by

$$
u_{\infty}(x)=M \mathrm{e}^{-V(x)}, \quad M=\left(\int_{\mathbb{R}^{d}} \mathrm{e}^{-V(y)} \mathrm{d} y\right)^{-1} .
$$

A typical choice of the potential is

$$
V(x)=\frac{1}{2} m|x|^{2}, \quad m>0,
$$

which corresponds to kinetic energy when regarding $x$ as the velocity of the particle. In this case the steady state becomes a Gaussian.

Assuming that $u_{0}$ is a probability density we aim at showing that the solution of the initialvalue problem (1), (2) tends to $u_{\infty}$ in $L^{1}\left(\mathbb{R}^{d}\right)$ as $t \rightarrow \infty$, and we are interested in convergence rates which are as precise as possible. We should point out that we do not address existence and regularity of solutions to (1) but perform formal calculations, which become rigorous provided the solution is sufficiently smooth (and satisfies a suitable growth condition for $|x| \rightarrow \infty$ ) or can be justified by appropriate regularization techniques.

As to existence and regularity of solutions to (1), we are not aware of any work where this specific problem has been studied. However, there is a theory of abstract evolutionary integral equations, see e.g. [20, 25, 26], which generalizes semigroup theory and can be applied to (1). For example, by the subordination principle from Prüss [20, Chapter 4], any result on the generation of a $C_{0}$-semigroup for the classical Fokker-Planck equation (5) yields a related well-posedness result for equation (1) if the kernel $k$ is of type $\mathcal{P C}$. The theory in [26] can be used to prove existence and uniqueness of a weak solution to (1) in the Hilbert space setting considered in Section 5 below. Higher regularity of the solution can be obtained by means of the results and techniques from [20, Chapter 2,3] (maximal regularity in Hölder spaces in time, classical solutions) and [25] (strong $L^{p}$-solutions), at least in the time-fractional case. An analogue of the celebrated theorem of Jordan, Kinderlehrer and Otto on the gradient flow structure of the classical Fokker-Planck equation in the Wasserstein space $\mathcal{P}_{2}\left(\mathbb{R}^{d}\right)([8])$ seems to be unknown for equation (1) and would be highly desirable.

For the classical Fokker-Planck equation (5) and certain variants of it (including nonlinear problems), there is a huge literature on convergence to equilibrium as $t \rightarrow \infty$, see e.g. $[1,9,15,21]$ and the references given therein. A very powerful technique in this context is the entropy method as presented, e.g., in [9]. The key idea consists in proving a differential inequality of the form

$$
\frac{\mathrm{d}}{\mathrm{d} t} H(u(t)) \leq-\kappa H(u(t)), \quad t>0,
$$

with $\kappa>0$, for a suitable relative entropy $H$ associated with the steady state $u_{\infty}$. Such a relative entropy is of the form

$$
H(u):=H_{\phi}(u)=\int_{\mathbb{R}^{d}} \phi\left(\frac{u(x)}{u_{\infty}(x)}\right) u_{\infty}(x) \mathrm{d} x, \quad u \in L^{1}\left(\mathbb{R}^{d}\right), u \geq 0,\|u\|_{1}=1,
$$


with generating function $\phi:[0, \infty) \rightarrow \mathbb{R}$, which is strictly convex and satisfies $\phi(1)=0$. Note that Jensen's inequality implies that $H(u) \geq 0$. An important example is given by

$$
\phi_{\beta}(x)=x^{\beta}-1-\beta(x-1), \quad 1<\beta \leq 2 .
$$

Inequality (9) then implies the exponential decay of the relative entropy,

$$
H(u(t)) \leq H(u(0)) \mathrm{e}^{-\kappa t}, \quad t>0,
$$

which together with a suitable Csizár-Kullback-Pinsker inequality (see Theorem 2.1 below) leads to an exponential decay estimate for $\left\|u(t)-u_{\infty}\right\|_{L^{1}\left(\mathbb{R}^{d}\right)}$ provided that $H(u(0))<\infty$. It is well known (see e.g. [1] and [9, Chapter 2]) that (9) with $\kappa=2 \lambda$ can be established for smooth potentials satisfying the convexity condition (also termed Bakry-Emery condition)

$$
\nabla^{2} V(x) \geq \lambda I, \quad x \in \mathbb{R}^{d},
$$

where $\lambda>0$ and $\nabla^{2} V$ denotes the Hessian of $V$. This is possible since condition (12) ensures the validity of certain convex Sobolev inequalities, in particular the logarithmic Sobolev inequality and a weighted Poincaré inequality.

Assuming that condition (12) is satisfied, the key idea of the entropy method in the non-local in time case is to derive an integro-differential inequality of the form

$$
\frac{\mathrm{d}}{\mathrm{d} t}\left(k *\left[\Phi-\Phi_{0}\right]\right)(t)+\mu \Phi(t) \leq 0, \quad t>0,
$$

where $\mu>0, \Phi_{0}=\Phi(0)$ and $\Phi(t)$ is a suitable function depending on a relative entropy, e.g. a power $\Phi(t)=H(u(t))^{1 / \beta}$ with $\beta \in[1,2]$. By the comparison principle (cf. [22, Section 2.3]), (13) then implies the estimate

$$
\Phi(t) \leq s_{\mu}(t) \Phi_{0},
$$

where $s_{\mu}$, the so-called relaxation function, solves the problem

$$
\frac{\mathrm{d}}{\mathrm{d} t}\left(k *\left[s_{\mu}-1\right]\right)(t)+\mu s_{\mu}(t) \leq 0, \quad t>0, \quad s_{\mu}(0)=1 .
$$

The large-time behaviour of the relaxation function depends heavily on the kernel $k$. Denoting by $\hat{k}$ the Laplace transform of $k$, it is known that $s_{\mu}(t) \rightarrow 0$ as $t \rightarrow \infty$ if and only if

$$
\lim _{\lambda \rightarrow 0+} \frac{1}{\lambda \hat{k}(\lambda)}=\infty
$$

This condition is satisfied for most of the important examples, in particular in the time-fractional case (3) for all $\alpha \in(0,1)$; here $\hat{k}(\lambda)=\lambda^{\alpha-1}, \operatorname{Re} \lambda>0$.

Our main result is the following. Here, by saying that the kernel $k$ is of type $\mathcal{P C}$ we mean that $k \in L_{l o c}^{1}\left(\mathbb{R}_{+}\right)$is nonnegative and nonincreasing, and that there exists a kernel $l \in L_{l o c}^{1}\left(\mathbb{R}_{+}\right)$ such that $k * l=1$ in $(0, \infty)$, cf. Section 2.1 .

Theorem 1.1 Let $k$ be a kernel of type $\mathcal{P C}$ and $V \in C^{2}\left(\mathbb{R}^{d}\right)$ satisfying condition (12) for some $\lambda>0$ and such that $e^{-V} \in L^{1}\left(\mathbb{R}^{d}\right)$. Let further $u_{0} \in L^{1}\left(\mathbb{R}^{d}\right)$ be a probability density. Defining $u_{\infty}$ as in (8) the following statements hold true. 
Part A (general entropy) Let $\phi$ be an admissible generating function according to Definition 2.1 and let $H(u)$ be the corresponding relative entropy associated with $u_{\infty}$ given by (10). Assume that $H\left(u_{0}\right)<\infty$ and that the positive solution $u$ of (1), (2) is sufficiently smooth. Then

$$
H(u(t)) \leq s_{2 \lambda}(t) H\left(u_{0}\right), \quad t>0 .
$$

Moreover,

$$
\left\|u(t)-u_{\infty}\right\|_{L^{1}\left(\mathbb{R}^{d}\right)} \leq C_{\phi} \sqrt{s_{2 \lambda}(t)} \sqrt{H\left(u_{0}\right)}, \quad t>0,
$$

with $C_{\phi}=\sqrt{2 / \phi^{\prime \prime}(1)}$. In particular, if in addition $k$ satisfies (16), then $u(t) \rightarrow u_{\infty}$ in $L^{1}\left(\mathbb{R}^{d}\right)$ as $t \rightarrow \infty$.

Part B (power type entropy) Let $\beta \in(1,2]$ and $\phi_{\beta}$ be defined as in (11). Let $H(u)$ be the corresponding relative entropy associated with $u_{\infty}$. Assume that $H\left(u_{0}\right)<\infty$ and that the positive solution $u$ of (1), (2) is sufficiently smooth. Then

$$
H(u(t)) \leq\left(s_{2 \lambda / \beta}(t)\right)^{\beta} H\left(u_{0}\right), \quad t>0 .
$$

Moreover,

$$
\left\|u(t)-u_{\infty}\right\|_{L^{1}\left(\mathbb{R}^{d}\right)} \leq \sqrt{\frac{2}{\beta(\beta-1)}}\left(s_{2 \lambda / \beta}(t)\right)^{\beta / 2} \sqrt{H\left(u_{0}\right)}, \quad t>0 .
$$

Part A of Theorem 1.1 says that, for any admissible entropy, (14) is true with $\Phi(t)=H(u(t))$ and $\mu=2 \lambda$. That is, under the additional assumption (16), the entropy decays at least as fast as the relaxation function $s_{2 \lambda}(t)$, and $\left\|u(t)-u_{\infty}\right\|_{L^{1}\left(\mathbb{R}^{d}\right)}$ is controlled by the square root of $s_{2 \lambda}(t)$. Note that Part A covers the important Boltzmann entropy defined by (34) in Section 2.3.

These results are the analogues of those for the classical Fokker-Planck equation (5), where the corresponding relaxation function solves the ODE

$$
\frac{\mathrm{d}}{\mathrm{d} t} s_{\mu}(t)+\mu s_{\mu}(t)=0, t>0, \quad s_{\mu}(0)=1,
$$

with $\mu=2 \lambda$, that is $s_{\mu}(t)=e^{-\mu t}=e^{-2 \lambda t}$. No matter which entropy is used, the norm $\left\|u(t)-u_{\infty}\right\|_{L^{1}\left(\mathbb{R}^{d}\right)}$ decays in the classical case (at least as fast) as $e^{-\lambda t}\left(=\sqrt{s_{2 \lambda}(t)}\right)$ (up to some constant).

As Part B of Theorem 1.1 shows, the latter is no longer the case in the non-local in time situation, in the sense that the decay rates improve for higher values of $\beta \in(1,2]$ when using the entropy generated by the power type function $\phi_{\beta}$. Observe that for $\beta=2$ we even reach the best possible decay estimate for $\left\|u(t)-u_{\infty}\right\|_{L^{1}\left(\mathbb{R}^{d}\right)}$ one can hope for, with a factor $s_{\lambda}(t)$ on the right-hand side of (20). In the classical case, we have the identity

$$
\left(s_{2 \lambda / \beta}(t)\right)^{\beta / 2}=\left(e^{-\frac{2 \lambda t}{\beta}}\right)^{\beta / 2}=e^{-\lambda t}=s_{\lambda}(t),
$$

which is not true any more in the non-local case, where, in general, $s_{\lambda}(t)$ decays faster than $\left(s_{2 \lambda / \beta}(t)\right)^{\beta / 2}$ for $\beta \in[1,2)$.

Notice as well that in case of the generating function $\phi_{\beta}$, the condition $H\left(u_{0}\right)<\infty$ is equivalent to

$$
\int_{\mathbb{R}^{d}}\left(\frac{u_{0}}{u_{\infty}}\right)^{\beta} u_{\infty} \mathrm{d} x<\infty
$$


which is stronger for larger values of $\beta$, by Hölder's inequality. Thus in contrast to the local Fokker-Planck equation, the decay rates improve with higher integrability of the initial datum w.r.t. the corresponding weighted Lebesgue space.

In the time-fractional case, the decay behaviour of the relaxation function can be quantified precisely. Theorem 1.1 leads to the following result.

Corollary 1.1 (fractional dynamics) Let $\alpha \in(0,1)$ and $k(t)=\frac{t^{-\alpha}}{\Gamma(1-\alpha)}, t>0$. Let $V, \lambda$, and $u_{\infty}$ be as in Theorem 1.1 and suppose that $u_{0} \in L^{1}\left(\mathbb{R}^{d}\right)$ is a probability density.

Part A (general entropy) Let $\phi$ be an admissible generating function and $H(u)$ be the corresponding relative entropy associated with $u_{\infty}$. Assume that $H\left(u_{0}\right)<\infty$ and that the positive solution $u$ of (1), (2) is sufficiently smooth. Then

$$
H(u(t)) \leq \frac{C(\alpha, \lambda)}{1+t^{\alpha}} H\left(u_{0}\right), \quad t>0
$$

and

$$
\left\|u(t)-u_{\infty}\right\|_{L^{1}\left(\mathbb{R}^{d}\right)} \leq \frac{C(\alpha, \lambda, \phi)}{1+t^{\alpha / 2}} \sqrt{H\left(u_{0}\right)}, \quad t>0 .
$$

Part B (power type entropy) Let $\beta \in(1,2]$ and $\phi_{\beta}$ be defined as in (11). Let $H(u)$ be the corresponding relative entropy. Assume that $H\left(u_{0}\right)<\infty$ and that the positive solution $u$ of (1), (2) is sufficiently smooth. Then

$$
H(u(t)) \leq \frac{C(\alpha, \beta, \lambda)}{1+t^{\alpha \beta}} H\left(u_{0}\right), \quad t>0,
$$

and

$$
\left\|u(t)-u_{\infty}\right\|_{L^{1}\left(\mathbb{R}^{d}\right)} \leq \frac{\tilde{C}(\alpha, \beta, \lambda)}{1+t^{\alpha \beta / 2}} \sqrt{H\left(u_{0}\right)}, \quad t>0 .
$$

Our proof of Theorem 1.1 makes heavily use of the fundamental identity (27) for the integrodifferential operator $B v=\partial_{t}(k * v)$. In order to obtain the improved estimates in the case of the power type entropy, we derive delicate estimates for the generating function $\phi_{\beta}$ and the associated entropy and employ the fundamental identity in its full strength, that is, all the terms on the right hand side of (27) are used. It is also noteworthy that we have to apply the fundamental identity twice.

It turns out that our method also applies to the time-discretized classical Fokker-Planck equation. Using our estimates for the generating function $\phi_{\beta}$ and the associated entropy we are seemingly able to improve existing results from the literature. The point is that in the time discrete case (where the equation is non-local as well), the decay rates become better with higher integrability of the initial datum, exactly as in Part B of Theorem 1.1. We strongly believe that our techniques may be also useful in the context of other time-discrete schemes for classical PDEs.

The paper is organized as follows. In Section 2 we collect some preliminaries on kernels of type $\mathcal{P C}$ and the associated relaxation functions, we recall the fundamental identity for operators of the form $\partial_{t}(k * \cdot)$, and we describe the class of admissible relative entropies and recall the general Csiszár-Kullback-Pinsker inequality as well as the convex Sobolev inequality. Section 3 contains the proof of our main result, Theorem 1.1. In Section 4 we discuss several important examples of kernels of type $\mathcal{P C}$, in particular we consider the time-fractional case and prove Corollary 1.1. In Section 5 we discuss the optimality of the entropy decay rate in the case $\beta=2$. Finally, Section 6 is devoted to the time-discrete case. 


\section{Preliminaries}

\subsection{Kernels of type $\mathcal{P C}$ and relaxation functions}

Throughout this paper we assume that the kernel $k$ in (1) is of type $\mathcal{P C}$. By this we mean that it satisfies the condition

$(\mathcal{P C}) k \in L_{l o c}^{1}\left(\mathbb{R}_{+}\right)$is nonnegative and nonincreasing, and there exists a kernel $l \in L_{\text {loc }}^{1}\left(\mathbb{R}_{+}\right)$such that $k * l=1$ in $(0, \infty)$.

In this case we also use the notation $(k, l) \in \mathcal{P C}$. Note that $(k, l) \in \mathcal{P C}$ implies that $l$ is nonnegative, cf. [3, Theorem 2.2].

Condition $(\mathcal{P C})$ has already been used before in a couple of papers (see e.g. $[10,22,23]$ ). It covers many important examples of integro-differential operators w.r.t. time that are used in physics to describe subdiffusion processes. An important example is given by $(k, l)=\left(g_{1-\alpha}, g_{\alpha}\right)$ with $\alpha \in(0,1)$, where $g_{\beta}$ denotes the standard kernel

$$
g_{\beta}(t)=\frac{t^{\beta-1}}{\Gamma(\beta)}, \quad t>0, \quad \beta>0
$$

In this case, the term $\partial_{t}(k * v)$ becomes the Riemann-Liouville fractional derivative $\partial_{t}^{\alpha} v$, and $k * \partial_{t} v={ }^{c} D_{t}^{\alpha} v$, the Caputo fractional derivative of order $\alpha$ (cf. [12]). We then also call (1) time-fractional Fokker-Planck equation.

Condition $(\mathcal{P C})$ also contains the multi-term fractional diffusion case, see Example 4.2 below. Another interesting and important class of examples is given by

$$
k(t)=\int_{0}^{1} g_{\beta}(t) \omega(\beta) \mathrm{d} \beta,
$$

where $\omega \in C([0,1])$ is a nonnegative weight function that does not vanish everywhere. In this situation the operator $\partial_{t}(k * \cdot)$ is a so-called operator of distributed order, and (1) is an example of an ultraslow Fokker-Planck equation if $\omega(0) \neq 0([13])$. As we shall see, the obtained decay rate for the entropy will partly justify the chosen name. The special case $\omega \equiv 1$ is discussed in Example 4.3 below.

Assuming $(k, l) \in \mathcal{P C}$ and $\mu \geq 0$ we recall that the associated relaxation function $s_{\mu}$ is defined via the problem (15). Convolving the first equation in (15) with $l$, it is easy to check that problem (15) is equivalent to the Volterra equation

$$
s_{\mu}(t)+\mu\left(l * s_{\mu}\right)(t)=1, \quad t \geq 0 .
$$

It is known that $s_{\mu}$ is nonnegative, nonincreasing, and that $s_{\mu} \in H_{l o c}^{1,1}\left(\mathbb{R}_{+}\right)$; furthermore $\partial_{\mu} s_{\mu}(t) \leq$ 0 , see e.g. Prüss [20]. Moreover, one knows that for any $\mu \geq 0$ there holds

$$
\frac{1}{1+\mu k(t)^{-1}} \leq s_{\mu}(t) \leq \frac{1}{1+\mu(1 * l)(t)}, \quad t>0,
$$

which also entails that

$$
\left[1-s_{\mu}(t)\right] k(t) \leq \mu s_{\mu}(t) \leq\left[1-s_{\mu}(t)\right] \frac{1}{(1 * l)(t)}, \quad t>0,
$$


see [22]. This implies that for any fixed $\mu>0, s_{\mu}(t)$ cannot decay faster than the kernel $k(t)$, and $s_{\mu}(t)$ decays at least like $(1 * l)(t)^{-1}$. Note that $\lim _{t \rightarrow \infty} s_{\mu}(t)=0$ if and only if $l \notin L^{1}\left(\mathbb{R}_{+}\right)$, see e.g. [22, Lemma 6.1]. Using

$$
\hat{k}(\lambda) \hat{l}(\lambda)=\frac{1}{\lambda}, \quad \lambda>0,
$$

it is easy to see that $l \notin L^{1}\left(\mathbb{R}_{+}\right)$if and only if condition (16) is satisfied.

\subsection{The fundamental identity for the operator $\partial_{t}(k * \cdot)$}

To simplify the notations, in what follows we use the symbol $\partial_{t}$ for the derivative also for the functions that depend only on $t$. An important tool in our approach is the so-called fundamental identity for integro-differential operators of the form $\partial_{t}(k * \cdot)$, cf. also [23, 24]. Let $k \in L_{l o c}^{1}\left(\mathbb{R}_{+}\right)$ be a nonnegative and nonincreasing kernel, $I \subset \mathbb{R}$ an interval, $\psi \in C^{1}(I)$, and $u \in L^{1}((0, T))$ with $u(t) \in I$ for a.a. $t \in(0, T)$. Then, for sufficiently smooth $u$ and $k$ there holds for a.a. $t \in(0, T)$

$$
\begin{aligned}
\psi^{\prime}(u(t)) \partial_{t}(k * u)(t) & =\partial_{t}(k * \psi(u))(t)+\left(-\psi(u(t))+\psi^{\prime}(u(t)) u(t)\right) k(t) \\
& +\int_{0}^{t}\left(\psi(u(t-s))-\psi(u(t))-\psi^{\prime}(u(t))[u(t-s)-u(t)]\right)\left(-k^{\prime}(s)\right) \mathrm{d} s
\end{aligned}
$$

This can be shown by a straightforward computation. Observe that the third term on the righthand side is nonnegative if $\psi$ is convex. Assuming in addition that $\psi$ is convex and that $u_{0} \in I$, it follows from (27) that

$$
\psi^{\prime}(u(t)) \partial_{t}\left(k *\left[u-u_{0}\right]\right)(t) \geq \partial_{t}\left(k *\left[\psi(u)-\psi\left(u_{0}\right)\right]\right)(t), \quad t \in(0, T),
$$

cf. [10, Corollary 6.1]. Note that here it is not assumed that $u_{0}=u(0)$.

We point out again that in this paper we perform formal calculations, that is, we assume that the solution is smooth enough so that (27) and (28) can be used with a kernel $k$ of type $\mathcal{P C}$, which is always singular at $t=0$. For less regular solutions (e.g. weak solutions in a certain sense), an important regularization technique consists in replacing the operator $\partial_{t}(k * \cdot)$ by its

Yosida approximations $\partial_{t}\left(k_{n} * \cdot\right), n \in \mathbb{N}$, with the more regular kernels $k_{n}=n s_{n} \in H_{l o c}^{1,1}\left(\mathbb{R}_{+}\right)$. We refer e.g. to $[22,23,24]$, where this method has been used to prove rigorous estimates in a weak setting.

The following technical lemma is an application of the convexity inequality (28). The function $w$ and the kernel $k$ involved have to be sufficiently regular so that (28) can be used.

Lemma 2.1 Let $T>0$ and $k \in L_{\text {loc }}^{1}\left(\mathbb{R}_{+}\right)$be a nonnegative and nonincreasing kernel. Let $w:[0, T] \rightarrow[0, \infty)$ be a sufficiently smooth function, $w_{0} \in[0, \infty)$ and $\delta, \gamma, \eta>0$ such that $\delta<\eta$. Let further $\mu \in L^{1}((0, T))$ and assume that

$$
w(t)^{\delta} \partial_{t}\left(k *\left[w^{\gamma}-w_{0}^{\gamma}\right]\right)(t)+\mu(t) w(t)^{\eta} \leq 0, \quad \text { a.a.t } \in(0, T) .
$$

Then there holds

$$
\partial_{t}\left(k *\left[w^{\gamma}-w_{0}^{\gamma}\right]\right)(t)+\mu(t) w(t)^{\eta-\delta} \leq 0, \quad \text { a.a.t } \in(0, T) .
$$


Proof. Set $v(t)=w(t)^{\gamma}, v_{0}=w_{0}^{\gamma}$, and define for $\varepsilon>0$ the function $\psi_{\varepsilon}:[0, \infty) \rightarrow[0, \infty)$ by

$$
\psi_{\varepsilon}(y)=\int_{0}^{y} \frac{r^{\delta / \gamma}}{r^{\delta / \gamma}+\varepsilon} \mathrm{d} r, \quad y \geq 0 .
$$

Clearly, $\psi_{\varepsilon} \in C^{1}([0, \infty))$ with

$$
\psi_{\varepsilon}^{\prime}(y)=\frac{y^{\delta / \gamma}}{y^{\delta / \gamma}+\varepsilon}=1-\frac{\varepsilon}{y^{\delta / \gamma}+\varepsilon}, \quad y \geq 0,
$$

and $\psi_{\varepsilon}$ is convex. Dividing (29) by $w(t)^{\delta}+\varepsilon$ we get

$$
\psi_{\varepsilon}^{\prime}(v(t)) \partial_{t}\left(k *\left[v-v_{0}\right]\right)(t)+\frac{\mu(t) w(t)^{\eta}}{w(t)^{\delta}+\varepsilon} \leq 0,
$$

which further yields

$$
\partial_{t}\left(k *\left[\psi_{\varepsilon}(v)-\psi_{\varepsilon}\left(v_{0}\right)\right]\right)(t)+\frac{\mu(t) w(t)^{\eta}}{w(t)^{\delta}+\varepsilon} \leq 0,
$$

by the convexity inequality (28).

Next, let $\varphi \in C_{0}^{\infty}((0, T))$ be a nonnegative test function. Multiplying the last inequality by $\varphi$, integrating over $(0, T)$ and integrating by parts gives

$$
\int_{0}^{T}\left(-\left(k *\left[\psi_{\varepsilon}(v)-\psi_{\varepsilon}\left(v_{0}\right)\right]\right)(t) \partial_{t} \varphi(t)+\frac{\mu(t) w(t)^{\eta}}{w(t)^{\delta}+\varepsilon} \varphi(t)\right) \mathrm{d} t \leq 0 .
$$

Sending $\varepsilon \rightarrow 0+$ and using that $\lim _{\varepsilon \rightarrow 0+} \psi_{\varepsilon}(y)=y$ for all $y \geq 0$ as well as

$$
\left|\psi_{\varepsilon}(v(t))-\psi_{\varepsilon}\left(v_{0}\right)\right| \leq \psi_{\varepsilon}(v(t))+\psi_{\varepsilon}\left(v_{0}\right) \leq v(t)+v_{0}, \quad t \in[0, T]
$$

and

$$
\left|\frac{\mu(t) w(t)^{\eta}}{w(t)^{\delta}+\varepsilon}\right| \leq|\mu(t)| w(t)^{\eta-\delta}, \quad \text { a.a. } t \in(0, T),
$$

we then obtain by Lebesgue's theorem on dominated convergence that

$$
\int_{0}^{T}\left(-\left(k *\left[v-v_{0}\right]\right)(t) \partial_{t} \varphi(t)+\mu(t) w(t)^{\eta-\delta} \varphi(t)\right) \mathrm{d} t \leq 0,
$$

and thus

$$
\int_{0}^{T}\left(\partial_{t}\left(k *\left[v-v_{0}\right]\right)(t)+\mu(t) w(t)^{\eta-\delta}\right) \varphi(t) \mathrm{d} t \leq 0,
$$

for all nonnegative test functions $\varphi$. The assertion of the lemma follows now by the fundamental lemma of the calculus of variations. 


\subsection{Admissible relative entropies and related inequalities}

Recall that a relative entropy $H(u)$ is induced by a generating function $\phi$, cf. (10). In this subsection we describe the class of admissible functions $\phi$ and discuss some important examples. The following definition has been taken from [1].

Definition 2.1 Let $I=(0, \infty)$ and $\phi \in C(\bar{I}) \cap C^{4}(I)$ satisfy the conditions

$$
\begin{aligned}
\phi(1) & =0 \\
\phi^{\prime \prime} \not \equiv 0, \quad \phi^{\prime \prime} & \geq 0 \quad \text { on } I, \\
\left(\phi^{(3)}\right)^{2} & \leq \frac{1}{2} \phi^{\prime \prime} \phi^{(4)} \quad \text { on } I .
\end{aligned}
$$

Let $u_{1}, u_{2} \in L^{1}\left(\mathbb{R}^{d}\right)$ be positive functions with $\int_{\mathbb{R}^{d}} u_{1} \mathrm{~d} x=\int_{\mathbb{R}^{d}} u_{2} \mathrm{~d} x=1$. Then

$$
E_{\phi}\left(u_{1} \mid u_{2}\right)=\int_{\mathbb{R}^{d}} \phi\left(\frac{u_{1}(x)}{u_{2}(x)}\right) u_{2}(x) \mathrm{d} x
$$

is called an admissible relative entropy of $u_{1}$ with respect to $u_{2}$ with generating function $\phi$.

Comparing this definition with (10), we see that the relative entropies considered in this paper are of the form

$$
H(u)=H_{\phi}(u)=E_{\phi}\left(u \mid u_{\infty}\right),
$$

where $u_{\infty}$ is the unique equilibrium of (1) given in (8).

Important examples of admissible generating functions are the power type function

$$
\phi_{\beta}(x)=x^{\beta}-1-\beta(x-1), \quad 1<\beta \leq 2,
$$

and

$$
\phi(x)=x(\log (x)-1)+1 .
$$

Note that the former takes a simple form in the special case $\beta=2$. In fact,

$$
\phi_{2}(x)=x^{2}-1-2(x-1)=(x-1)^{2} .
$$

The logarithmic function (33) leads to the Boltzmann entropy

$$
H(u)=\int_{\mathbb{R}^{d}} u \log \left(\frac{u}{u_{\infty}}\right) \mathrm{d} x
$$

of the probability density $u$ and can be viewed as a limiting case of $\phi_{\beta}$ as $\beta \rightarrow 1+$ in the sense that

$$
\lim _{\beta \rightarrow 1+} \frac{\phi_{\beta}(x)}{\beta-1}=x(\log (x)-1)+1 .
$$

The following inequality provides a control of the $L^{1}$ distance of two probability densities $f, g$ by the relative entropy $E_{\phi}(f \mid g)$. It can be found in [1, Section 2.2], see also [9, Theorem A.3].

Theorem 2.1 (General Csiszár-Kullback-Pinsker inequality) Let $f, g \in L^{1}\left(\mathbb{R}^{d}\right)$ be positive functions with unit mass. If $\phi$ satisfies the assumptions of Definition 2.1, then

$$
\|f-g\|_{L^{1}\left(\mathbb{R}^{d}\right)}^{2} \leq \frac{2}{\phi^{\prime \prime}(1)} \int_{\mathbb{R}^{d}} \phi\left(\frac{f}{g}\right) g \mathrm{~d} x .
$$


We also need the following version of the convex Sobolev inequality (see [9, Corollary 2.1]).

Theorem 2.2 (Convex Sobolev inequality) Let $\phi$ be an admissible generating function in the sense of Definition 2.1 and assume that $V \in C^{2}\left(\mathbb{R}^{d}\right)$ satisfies the condition (12) for some $\lambda>0$ and is such that $e^{-V} \in L^{1}\left(\mathbb{R}^{d}\right)$. Defining $u_{\infty}$ as in (8) there holds

$$
\int_{\mathbb{R}^{d}} \phi\left(\frac{u}{u_{\infty}}\right) u_{\infty} \mathrm{d} x \leq \frac{1}{2 \lambda} \int_{\mathbb{R}^{d}} \phi^{\prime \prime}\left(\frac{u}{u_{\infty}}\right)\left|\nabla \frac{u}{u_{\infty}}\right|^{2} u_{\infty} \mathrm{d} x
$$

for all nonnegative integrable functions u for which the integrals are defined.

\section{Proof of Theorem 1.1}

\subsection{Part A - general entropies}

Let the assumptions of Theorem 1.1 (Part A) be satisfied. We set

$$
\mathcal{L}^{*} u:=\Delta u+\nabla \cdot(u \nabla V) .
$$

Observe that

$$
\nabla u_{\infty}=-u_{\infty} \nabla V
$$

and thus

$$
\nabla \cdot\left(u_{\infty} \nabla\left(\frac{u}{u_{\infty}}\right)\right)=\nabla \cdot\left(\nabla u+u_{\infty} u\left(-\frac{1}{u_{\infty}^{2}}\right) \nabla u_{\infty}\right)=\mathcal{L}^{*} u
$$

Setting

$$
v:=\frac{u}{u_{\infty}}, \quad v_{0}:=\frac{u_{0}}{u_{\infty}}
$$

equation (1) can be rewritten as

$$
\partial_{t}\left(k *\left[v-v_{0}\right]\right) u_{\infty}-\nabla \cdot\left(u_{\infty} \nabla v\right)=0
$$

Multiplying (37) by $\phi^{\prime}(v)$, integrating over $\mathbb{R}^{d}$ and integrating by parts we obtain

$$
\int_{\mathbb{R}^{d}} \phi^{\prime}(v) \partial_{t}\left(k *\left[v-v_{0}\right]\right) u_{\infty} \mathrm{d} x=-\int_{\mathbb{R}^{d}} \phi^{\prime \prime}(v)|\nabla v|^{2} u_{\infty} \mathrm{d} x .
$$

The right-hand side of (38) can be estimated from above by means of the convex Sobolev inequality, Theorem 2.2. This gives

$$
\int_{\mathbb{R}^{d}} \phi^{\prime}(v) \partial_{t}\left(k *\left[v-v_{0}\right]\right) u_{\infty} \mathrm{d} x \leq-2 \lambda \int_{\mathbb{R}^{d}} \phi(v) u_{\infty} \mathrm{d} x=-2 \lambda H(u) .
$$

For the left-hand side of (39) we use the fundamental identity in the form of the convexity inequality (28) ( $\phi$ is convex!), thereby obtaining

$$
\int_{\mathbb{R}^{d}} \partial_{t}\left(k *\left[\phi(v)-\phi\left(v_{0}\right)\right]\right) u_{\infty} \mathrm{d} x \leq-2 \lambda H(u),
$$


which is equivalent to

$$
\partial_{t}\left(k *\left[H(u)-H\left(u_{0}\right)\right]\right)+2 \lambda H(u) \leq 0 .
$$

The first assertion from Part A, estimate (17), follows now from (40) by the comparison principle (cf. [22, Section 2.3]).

The $L^{1}$-estimate (18) is a consequence of (17) and the Csiszár-Kullback-Pinsker inequality stated in Theorem 2.1. This finishes the proof of Part A of Theorem 1.1.

\subsection{Part B - power type entropy}

The proof of Part B is much more involved. In order to obtain the desired inequality (19) one also has to exploit the third term in the fundamental identity (27).

The basic idea of the proof is inspired by [22, Lemma 3.1]. We split the left-hand side of (38) by writing

$$
\begin{aligned}
\int_{\mathbb{R}^{d}} \phi^{\prime}(v) \partial_{t}\left(k *\left[v-v_{0}\right]\right) u_{\infty} \mathrm{d} x & =\int_{\mathbb{R}^{d}} \phi^{\prime}(v) \partial_{t}(k * v) u_{\infty} \mathrm{d} x-\int_{\mathbb{R}^{d}} \phi^{\prime}(v) v_{0} u_{\infty} \mathrm{d} x k(t) \\
& =: I_{1}(t)-I_{2}(t) .
\end{aligned}
$$

Applying the fundamental identity (27) pointwise in $\mathbb{R}^{d}$ we have at time $t>0$

$$
\begin{aligned}
I_{1}(t)= & \int_{\mathbb{R}^{d}} \partial_{t}(k * \phi(v)) u_{\infty} \mathrm{d} x+\int_{\mathbb{R}^{d}}\left(-\phi(v)+\phi^{\prime}(v) v\right) u_{\infty} \mathrm{d} x k(t) \\
& +\int_{\mathbb{R}^{d}} \int_{0}^{t}\left(\phi(v(t-s))-\phi(v(t))-\phi^{\prime}(v(t))[v(t-s)-v(t)]\right)\left(-k^{\prime}(s)\right) \mathrm{d} s u_{\infty} \mathrm{d} x .
\end{aligned}
$$

Note that $v$ depends both on $t$ and $x$ although we have used the notation $v(t)$. Since (42) contains different time levels and the formula is quite long, it is more convenient to adopt the shorthand notation $v(t)$ and $u(t)$ instead of $v(t, x)$ and $u(t, x)$, respectively.

The first term on the right hand side of (42) is simply

$$
\partial_{t}(k * H(u))(t),
$$

in view of the definition of the relative entropy (10).

For the second term we use conservation of mass, which gives

$$
\int_{\mathbb{R}^{d}} v(t) u_{\infty} \mathrm{d} x=\int_{\mathbb{R}^{d}} u(t) \mathrm{d} x=\int_{\mathbb{R}^{d}} u_{\infty} \mathrm{d} x=1 .
$$

This allows to add arbitrary multiples of $v-1$ inside the spatial integral with weight function $u_{\infty}$. Recalling that

$$
\phi(x)=\phi_{\beta}(x)=x^{\beta}-1-\beta(x-1), \quad 1<\beta \leq 2,
$$

we thus have at time $t>0$

$$
\begin{aligned}
\int_{\mathbb{R}^{d}}(-\phi(v) & \left.+\phi^{\prime}(v) v\right) u_{\infty} \mathrm{d} x k(t)=\int_{\mathbb{R}^{d}}\left(-\phi(v)+\beta\left(v^{\beta-1}-1\right) v\right) u_{\infty} \mathrm{d} x k(t) \\
& =-H(u(t)) k(t)+\beta \int_{\mathbb{R}^{d}}\left(\left(v^{\beta}-v\right)-(\beta-1)[v-1]\right) u_{\infty} \mathrm{d} x k(t) \\
& =(\beta-1) H(u(t)) k(t) .
\end{aligned}
$$


The third term on the right-hand side of (42) is the most difficult one to handle. First of all, we have

$$
\begin{aligned}
& \int_{\mathbb{R}^{d}} \int_{0}^{t}\left(\phi(v(t-s))-\phi(v(t))-\phi^{\prime}(v(t))[v(t-s)-v(t)]\right)\left(-k^{\prime}(s)\right) \mathrm{d} s u_{\infty} \mathrm{d} x \\
= & \int_{0}^{t}(H(u(t-s))-H(u(t)))\left(-k^{\prime}(s)\right) \mathrm{d} s \\
& -\beta \int_{0}^{t} \int_{\mathbb{R}^{d}}\left(v(t)^{\beta-1}-1\right)[v(t-s)-v(t)] u_{\infty} \mathrm{d} x\left(-k^{\prime}(s)\right) \mathrm{d} s .
\end{aligned}
$$

The first integral on the right hand side is already expressed in terms of the entropy, so this is a good term and we are left with the second integral. Set

$$
I_{3}(t, s)=\int_{\mathbb{R}^{d}}\left(v(t)^{\beta-1}-1\right)[v(t-s)-v(t)] u_{\infty} \mathrm{d} x .
$$

Using again conservation of mass, we have

$$
\begin{aligned}
I_{3}(t, s)= & \int_{\mathbb{R}^{d}}\left(v(t)^{\beta-1} v(t-s)-v(t)^{\beta}-v(t-s)+v(t)\right) u_{\infty} \mathrm{d} x \\
= & -\int_{\mathbb{R}^{d}}\left(v(t)^{\beta}-1-\beta[v(t)-1]\right) u_{\infty} \mathrm{d} x \\
& +\int_{\mathbb{R}^{d}}\left(-(\beta-1)[v(t)-1]+v(t)^{\beta-1} v(t-s)-v(t-s)\right) u_{\infty} \mathrm{d} x \\
= & -H(u(t))+\int_{\mathbb{R}^{d}}\left(v(t)^{\beta-1} v(t-s)-v(t)\right) u_{\infty} \mathrm{d} x .
\end{aligned}
$$

The last integral can be estimated from above by an expression which only involves two entropy terms. Indeed we can prove the following result.

Lemma 3.1 Let $1<\beta \leq 2$. Then for the entropy (10) with $\phi:=\phi_{\beta}$ given by (11) there holds

$$
\int_{\mathbb{R}^{d}}\left(v(t)^{\beta-1} v(t-s)-v(t)\right) u_{\infty} \mathrm{d} x \leq H(u(t))^{\frac{\beta-1}{\beta}} H(u(t-s))^{\frac{1}{\beta}}
$$

Before entering the proof we make a couple of remarks. Due to conservation of mass we can add arbitrary multiples of $(v(t)-1) u_{\infty}$ and $(v(t-s)-1) u_{\infty}$ to the integrand on the left-hand side of (43) without changing the value of the integral. The idea is to use Hölder's inequality but its direct use is not possible. So we have to modify the left-hand side of (43) appropriately. It turns out that it is useful to rewrite the left-hand side in the form

$$
\begin{aligned}
& \int_{\mathbb{R}^{d}}\left(v(t)^{\beta-1} v(t-s)-v(t)+(2-\beta)[v(t)-1]-[v(t-s)-1]\right) u_{\infty} \mathrm{d} x \\
= & \left.\int_{\mathbb{R}^{d}}\left(v(t)^{\beta-1} v(t-s)+(1-\beta) v(t)-v(t-s)+\beta-1\right)\right) u_{\infty} \mathrm{d} x .
\end{aligned}
$$

Then to show (43) we use the following pointwise estimate. 
Lemma 3.2 Let $1<\beta \leq 2$. Then for the function $\phi:=\phi_{\beta}$ defined by (11) there holds

$$
x^{\beta-1} y+(1-\beta) x-y+\beta-1 \leq \phi(x)^{\frac{\beta-1}{\beta}} \phi(y)^{\frac{1}{\beta}}, \quad x, y \geq 0 .
$$

Proof. We distinguish different cases. We begin with $\beta=2$. In this case the generating function is simply

$$
\phi(x)=(x-1)^{2} .
$$

On the other hand, the left-hand side of (44) is

$$
x y-x-y-1=(x-1)(y-1) \leq|x-1||y-1|,
$$

and so (44) clearly holds.

We consider now the case $1<\beta<2$. The left-hand side of (44) can be written in the form

$$
\left(x^{\beta-1}-1\right) y+(1-\beta)(x-1),
$$

which is, for any fixed $x \neq 1$, a first order polynomial in $y$. If $x=1$, the expression in (45) vanishes and the desired inequality is trivially true. We now distinguish the two cases $x<1$ and $x>1$.

The case $x<1$. In this case (45) is strictly decreasing in $y$, so obviously for large values of $y$ it is negative and we are done. We have

$$
\left(x^{\beta-1}-1\right) y+(1-\beta)(x-1) \leq 0 \Leftrightarrow y \geq \frac{(\beta-1)(1-x)}{1-x^{\beta-1}} .
$$

The function $f(x)=x^{\beta-1}-1$ is strictly concave and negative for $x<1$, and so there holds

$$
\frac{(\beta-1)(1-x)}{1-x^{\beta-1}}=(\beta-1) \frac{1-x}{f(1)-f(x)}<\frac{\beta-1}{f^{\prime}(1)}=1 .
$$

Hence it remains to prove the estimate (44) for $y<1$.

Assuming $y<1$ we study carefully the behaviour of the function

$$
F(x, y)=\phi(x)^{\frac{\beta-1}{\beta}} \phi(y)^{\frac{1}{\beta}}-\left(x^{\beta-1} y+(1-\beta) x-y+\beta-1\right) .
$$

On the diagonal $y=x$ the function $F$ is zero:

$$
\begin{aligned}
F(x, x) & =\phi(x)^{\frac{\beta-1}{\beta}} \phi(x)^{\frac{1}{\beta}}-\left(x^{\beta}+(1-\beta) x-x+\beta-1\right) \\
& =\phi(x)-\left(x^{\beta}-1-\beta(x-1)\right)=0 .
\end{aligned}
$$

We will prove that $F(x, y)>0$ whenever $x \neq y$. Let us first calculate the critical points of $F(x, \cdot)$ with $x<1$ being fixed. We have

$$
\frac{\partial F}{\partial y}=\phi(x)^{\frac{\beta-1}{\beta}} \phi(y)^{\frac{1-\beta}{\beta}}\left(y^{\beta-1}-1\right)-\left(x^{\beta-1}-1\right),
$$

which is clearly zero, if $y=x$. To prove that there are no other zeros, it is enough to show that $F(x, \cdot)$ is strictly convex. To this end we calculate the second derivative

$$
\frac{\partial^{2} F}{\partial y^{2}}=\phi(x)^{\frac{\beta-1}{\beta}} \phi(y)^{\frac{1-2 \beta}{\beta}}(\beta-1)\left(\phi(y) y^{\beta-2}-\left(y^{\beta-1}-1\right)^{2}\right) .
$$


The prefactors are positive, so it remains to study the function

$$
g(y)=\phi(y) y^{\beta-2}-\left(y^{\beta-1}-1\right)^{2}=(\beta-1) y^{\beta-2}+(2-\beta) y^{\beta-1}-1 .
$$

The first derivative of $g$ is

$$
g^{\prime}(y)=(2-\beta)(\beta-1) y^{\beta-3}(y-1),
$$

which is negative for $y<1$ and so $g$ is strictly decreasing. Since $g(1)=0$, we see that

$$
\frac{\partial^{2} F}{\partial y^{2}}>0
$$

whence $F(x, \cdot)$ is strictly convex for $y<1$ with $x<1$ being fixed arbitrarily. Therefore $F$ is zero only on the diagonal and positive outside the diagonal and we are done.

The case $x>1$. In this case (45) is strictly increasing in $y$, whence the left-hand side of (44) is nonpositive for all

$$
y \leq \frac{(\beta-1)(x-1)}{x^{\beta-1}-1} .
$$

Once again, since $f(x)=x^{\beta-1}-1$ is strictly concave and $x>1$, we have

$$
\frac{(\beta-1)(x-1)}{x^{\beta-1}-1}>\frac{\beta-1}{f^{\prime}(1)}=1
$$

and thus it remains to prove (44) for $y>1$. In this case $g$ defined by (47) is strictly increasing, by (48). Since $g(1)=0$, we have

$$
\frac{\partial^{2} F}{\partial y^{2}}>0
$$

whenever $y>1$. Therefore $F(x, \cdot)$ is again strictly convex for $y>1$ with $x>1$ being fixed arbitrarily, which completes the proof.

Now we can proceed to the proof of Lemma 3.1.

Proof of Lemma 3.1. By Lemma 3.2 and the remarks prior to it we may estimate as

$$
\int_{\mathbb{R}^{d}}\left(v(t)^{\beta-1} v(t-s)-v(t)\right) u_{\infty} \mathrm{d} x \leq \int_{\mathbb{R}^{d}} \phi(v(t))^{\frac{\beta-1}{\beta}} \phi(v(t-s))^{\frac{1}{\beta}} u_{\infty} \mathrm{d} x .
$$

The asserted inequality follows then by an application of Hölder's inequality with the exponents $p=\frac{\beta}{\beta-1}$ and $p^{\prime}=\beta$ with respect to the measure $\mu(\mathrm{d} x)=u_{\infty}(x) \mathrm{d} x$.

Remark 3.1 Observe that in the proof of Lemma 3.1 we only used the properties that $u(t)=$ $u(t, \cdot), u(t-s)=u(t-s, \cdot)$ and $u_{\infty}$ are probability densities on $\mathbb{R}^{d}$ with $u_{\infty}>0$ everywhere and that $v(t)=u(t) / u_{\infty}$ as well as $v(t-s)=u(t-s) / u_{\infty}$. Thus the argument yields a more general result. For example, it shows that if $f_{1}, f_{2}, g$ are probability densities on $\mathbb{R}^{d}$ with $g>0$ on $\mathbb{R}^{d}$ then we have with $h_{i}:=f_{i} / g, i=1,2$,

$$
\int_{\mathbb{R}^{d}} h_{1}^{\beta-1} h_{2} g \mathrm{~d} x-1=\int_{\mathbb{R}^{d}}\left(h_{1}^{\beta-1} h_{2}-h_{1}\right) g \mathrm{~d} x \leq E_{\phi_{\beta}}\left(f_{1} \mid g\right)^{\frac{\beta-1}{\beta}} E_{\phi_{\beta}}\left(f_{2} \mid g\right)^{\frac{1}{\beta}},
$$

where $\phi_{\beta}$ is as in Lemma 3.1, cf. (31) for the definition of the relative entropy. Evidently, as the above proof shows, this statement can be generalized further to more general probability spaces. 
Having Lemma 3.1 at our disposal we can estimate the term $I_{3}(t, s)$ from above by entropy terms as follows:

$$
I_{3}(t, s) \leq-H(u(t))+H(u(t))^{\frac{\beta-1}{\beta}} H(u(t-s))^{\frac{1}{\beta}} .
$$

Combining this estimate and the above calculations concerning the term $I_{1}(t)$ we obtain for $t>0$

$$
\begin{aligned}
I_{1}(t)= & \partial_{t}(k * H(u))(t)+(\beta-1) H(u(t)) k(t) \\
& +\int_{0}^{t}(H(u(t-s))-H(u(t)))\left(-k^{\prime}(s)\right) \mathrm{d} s-\beta \int_{0}^{t} I_{3}(t, s)\left(-k^{\prime}(s)\right) \mathrm{d} s \\
\geq & \partial_{t}(k * H(u))(t)+(\beta-1) H(u(t)) k(t) \\
& +\int_{0}^{t}\left(H(u(t-s))+(\beta-1) H(u(t))-\beta H(u(t))^{\frac{\beta-1}{\beta}} H(u(t-s))^{\frac{1}{\beta}}\right)\left(-k^{\prime}(s)\right) \mathrm{d} s \\
= & I_{4}(t) .
\end{aligned}
$$

A key idea is now to apply the fundamental identity a second time (!) to show that

$$
I_{4}(t)=\beta H(u(t))^{\frac{\beta-1}{\beta}} \partial_{t}\left(k * H(u)^{\frac{1}{\beta}}\right)(t) .
$$

In fact, setting

$$
w(t)=H(u(t))^{\frac{1}{\beta}}
$$

the fundamental identity (27) with $\psi(y)=y^{\beta}$ gives

$$
\begin{aligned}
\beta H(u(t))^{\frac{\beta-1}{\beta}} \partial_{t}\left(k * H(u)^{\frac{1}{\beta}}\right)(t)=\beta w(t)^{\beta-1} \partial_{t}(k * w)(t) \\
=\partial_{t}\left(k * w^{\beta}\right)(t)+(\beta-1) w(t)^{\beta} k(t) \\
\quad+\int_{0}^{t}\left(w(t-s)^{\beta}-w(t)^{\beta}-\beta w(t)^{\beta-1}[w(t-s)-w(t)]\right)\left(-k^{\prime}(s)\right) \mathrm{d} s \\
=\partial_{t}(k * H(u))(t)+(\beta-1) H(u(t)) k(t) \\
\quad+\int_{0}^{t}\left(H(u(t-s))-H(u(t))-\beta H(u(t))^{\frac{\beta-1}{\beta}}\left[H(u(t-s))^{\frac{1}{\beta}}-H(u(t))^{\frac{1}{\beta}}\right]\right)\left(-k^{\prime}(s)\right) \mathrm{d} s \\
=I_{4}(t) .
\end{aligned}
$$

Combining (49) and $I_{1}(t) \geq I_{4}(t)$ we obtain

$$
I_{1}(t) \geq \beta H(u(t))^{\frac{\beta-1}{\beta}} \partial_{t}\left(k * H(u)^{\frac{1}{\beta}}\right)(t) .
$$

It remains to estimate the term $I_{2}(t)$, which contains the initial datum. Using again conservation of mass we have

$$
I_{2}(t)=\int_{\mathbb{R}^{d}} \beta\left(v(t)^{\beta-1}-1\right) v_{0} u_{\infty} \mathrm{d} x k(t)=\beta \int_{\mathbb{R}^{d}}\left(v(t)^{\beta-1} v_{0}-v(t)\right) u_{\infty} \mathrm{d} x k(t) .
$$

By virtue of Remark 3.1 it follows that

$$
I_{2}(t) \leq \beta k(t) H(u(t))^{\frac{\beta-1}{\beta}} H\left(u_{0}\right)^{\frac{1}{\beta}} .
$$


Combining (39), (41), (50), (51) we obtain

$$
\beta H(u(t))^{\frac{\beta-1}{\beta}} \partial_{t}\left(k *\left(H(u)^{1 / \beta}-H\left(u_{0}\right)^{1 / \beta}\right)\right)(t) \leq-2 \lambda H(u(t)), \quad t>0 .
$$

Invoking Lemma 2.1 it follows from (52) that

$$
\partial_{t}\left(k *\left(H(u)^{1 / \beta}-H\left(u_{0}\right)^{1 / \beta}\right)\right)(t) \leq-\frac{2 \lambda}{\beta} H(u(t))^{1 / \beta},
$$

which in turn implies the asserted estimate (19), by the comparison principle (cf. [22, Section 2.3]). The second estimate in Part B, inequality (20), follows directly from (19) by the CsiszárKullback-Pinsker inequality stated in Theorem 2.1 with $\phi=\phi_{\beta}$. This finishes the proof of Part B of Theorem 1.1.

\section{Examples and proof of Corollary 1.1}

In this section we consider several important examples of kernels of type $\mathcal{P C}$ and look the long-time behaviour of the corresponding relaxation functions $s_{\mu}$. For more details and further examples we refer to [22, Section 6].

Example 4.1 The time-fractional case. We consider the pair

$$
(k, l)=\left(g_{1-\alpha}, g_{\alpha}\right),
$$

where $\alpha \in(0,1)$ and $g_{\beta}$ is defined by (25). Recall that the Laplace transform of $g_{\beta}, \beta>0$, is given by $\widehat{g_{\beta}}(z)=z^{-\beta}$, Re $z>0$, so it is easy to see that $g_{\beta_{1}} * g_{\beta_{2}}=g_{\beta_{1}+\beta_{2}}$ for all $\beta_{1}, \beta_{2}>0$. This implies

$$
(k * l)(t)=\left(g_{1-\alpha} * g_{\alpha}\right)(t)=g_{1}(t)=1, \quad t>0,
$$

and so it is clear that $\left(g_{1-\alpha}, g_{\alpha}\right) \in \mathcal{P C}$. We further have

$$
(1 * l)(t)=\left(1 * g_{\alpha}\right)(t)=g_{1+\alpha}(t)=\frac{t^{\alpha}}{\Gamma(1+\alpha)},
$$

which together with (26) shows that for $\mu \geq 0$ the corresponding relaxation function $s_{\mu}$ satisfies

$$
\frac{1}{1+\mu \Gamma(1-\alpha) t^{\alpha}} \leq s_{\mu}(t) \leq \frac{1}{1+\mu[\Gamma(1+\alpha)]^{-1} t^{\alpha}}, \quad t \geq 0 .
$$

Corollary 1.1 now follows from Theorem 1.1 and (54).

We remark that

$$
s_{\mu}(t)=E_{\alpha}\left(-\mu t^{\alpha}\right), \quad \text { where } E_{\alpha}(z):=\sum_{j=0}^{\infty} \frac{z^{j}}{\Gamma(\alpha j+1)}, z \in \mathbb{C},
$$

is the well-known Mittag-Leffler function (see e.g. [12]).

If one replaces the above pair of kernels by

$$
k(t)=g_{1-\alpha}(t) e^{-\gamma t}, \quad l(t)=g_{\alpha}(t) e^{-\gamma t}+\gamma\left(1 *\left[g_{\alpha} e^{-\gamma \cdot}\right]\right)(t), \quad t>0,
$$

with $\alpha \in(0,1)$ and $\gamma>0$, then again $(k, l) \in \mathcal{P C}$ and the associated relaxation function decays exponentially, see [22, Example 6.2]. 
Example 4.2 Multiterm fractional derivative. We consider the sum of two fractional derivatives. Let $0<\alpha<\beta<1$ and

$$
k(t)=g_{1-\alpha}(t)+g_{1-\beta}(t), \quad t>0 .
$$

Clearly, $k$ is completely monotone and $k(0+)=\infty$. It follows by Theorem 5.4 in Chapter 5 of [6] that the kernel $k$ has a resolvent $l \in L_{l o c}^{1}\left(\mathbb{R}_{+}\right)$of the first kind, that is $k * l=1$ on $(0, \infty)$, and this resolvent is completely monotone as well. In particular $(k, l) \in \mathcal{P C}$. Since

$$
\widehat{1 * l}(z)=\frac{1}{z} \frac{1}{z^{\alpha}+z^{\beta}} \sim \frac{1}{z^{1+\alpha}} \quad \text { as } z \rightarrow 0,
$$

the Karamata-Feller Tauberian theorem (see [5]) implies that $(1 * l)(t) \sim g_{1+\alpha}(t)$ as $t \rightarrow \infty$. On the other hand, $k(t) \sim g_{1-\alpha}(t)$ as $t \rightarrow \infty$, and so, using (26), we see that the relaxation function $s_{\mu}(t)$ has the same algebraic decay (as $t \rightarrow \infty$ ) as in Example 4.1; the decay rate is determined by the fractional derivative of lower order.

These considerations extend trivally to kernels $k(t)=\sum_{j=1}^{m} \delta_{j} g_{1-\alpha_{j}}(t)$ with $\delta_{j}>0$ and $0<\alpha_{1}<\alpha_{2}<\ldots<\alpha_{m}<1$.

Example 4.3 The distributed order case (an example of ultraslow diffusion). We consider the pair

$$
k(t)=\int_{0}^{1} g_{\beta}(t) \mathrm{d} \beta, \quad l(t)=\int_{0}^{\infty} \frac{e^{-s t}}{1+s} \mathrm{~d} s, \quad t>0 .
$$

Both kernels are nonnegative and nonincreasing, and there holds (see [22, Example 6.5])

$$
\hat{k}(z)=\frac{z-1}{z \log z}, \quad \hat{l}(z)=\frac{\log z}{z-1}, \quad \operatorname{Re} z>0 .
$$

Thus $(k, l) \in \mathcal{P C}$. There exists a number $T_{1}>1$ such that

$$
\frac{1}{2 k(t)} \leq \log t \leq 2(1 * l)(t), \quad t \geq T_{1},
$$

see [22, Example 6.5]. In view of (26) this shows that for $\mu>0$ the relaxation function $s_{\mu}(t)$ can be estimated for large times from above and from below by terms of the form $c / \log t$ with some constant $c>0$. So we get a logarithmic decay, which is certainly slower than in the time-fractional case for any $\alpha \in(0,1)$.

\section{Optimality of the entropy decay rate in the case $\beta=2$}

We consider the situation of Part B in Theorem 1.1 with $\beta=2$. The goal of this section is to show that in this case the estimate (19) is in general the best possible one can have. Since

$$
H(u)=\int_{\mathbb{R}^{d}}\left(\frac{u}{u_{\infty}}-1\right)^{2} u_{\infty} \mathrm{d} x=\int_{\mathbb{R}^{d}}\left(u-u_{\infty}\right)^{2} u_{\infty}^{-1} \mathrm{~d} x
$$

and $u_{\infty} \in L^{1}\left(\mathbb{R}^{d}\right)$, the assumption $H\left(u_{0}\right)<\infty$ means exactly that $u_{0}$ belongs to the weighted $L^{2}$-space

$$
L^{2}\left(u_{\infty}^{-1}\right):=L^{2}\left(\mathbb{R}^{d} ; u_{\infty}^{-1}\right)=\left\{w: \mathbb{R}^{d} \rightarrow \mathbb{R} \text { measurable s.t. }\|w\|_{L^{2}\left(u_{\infty}^{-1}\right)}^{2}:=\int_{\mathbb{R}^{d}} w^{2} u_{\infty}^{-1} \mathrm{~d} x<\infty\right\} .
$$


The entropy decay estimate (19) can be written as

$$
\left\|u(t)-u_{\infty}\right\|_{L^{2}\left(u_{\infty}^{-1}\right)} \leq s_{\lambda}(t)\left\|u_{0}-u_{\infty}\right\|_{L^{2}\left(u_{\infty}^{-1}\right)}, \quad t>0 .
$$

In terms of $v:=u / u_{\infty}$ and $v_{0}:=u_{0} / u_{\infty}$ the estimate (19) takes the form

$$
\|v(t)-1\|_{L^{2}\left(u_{\infty}\right)} \leq s_{\lambda}(t)\left\|v_{0}-1\right\|_{L^{2}\left(u_{\infty}\right)}, \quad t>0
$$

with the weighted $L^{2}$-space $L^{2}\left(u_{\infty}\right):=L^{2}\left(\mathbb{R}^{d} ; u_{\infty}\right)$. The convex Sobolev inequality $(2.2)$ becomes the Poincaré inequality w.r.t. the measure $d \mu=u_{\infty} d x$ for the function $v-1$ (which has zero mean); more precisely,

$$
\int_{\mathbb{R}^{d}}(v-1)^{2} u_{\infty} \mathrm{d} x \leq \frac{1}{\lambda} \int_{\mathbb{R}^{d}}|\nabla v|^{2} u_{\infty} \mathrm{d} x
$$

Anagolously to the classical case, which is discussed e.g. in [19], $u$ solves the non-local FokkerPlanck equation (1) with initial value $u_{0}$ if and only if $v=u / u_{\infty}$ solves the non-local backward Kolmogorov equation

$$
\partial_{t}\left(k *\left[v-v_{0}\right]\right)-\mathcal{L} v=0, \quad t>0, x \in \mathbb{R}^{d},
$$

with initial value $v_{0}=u_{0} / u_{\infty}$, where the operator $\mathcal{L}$ is given by

$$
\mathcal{L} f=\Delta f-\nabla V \cdot \nabla f .
$$

It is known that the operator $\mathcal{L}$ is the $L^{2}\left(\mathbb{R}^{d}\right)$ adjoint of the Fokker-Planck operator $\mathcal{L}^{*}$ appearing in (1) (see (36) for its definition). Moreover, $\mathcal{L}$ is self-adjoint with respect to the weighted $L^{2}$ inner product

$$
(f, g)_{L^{2}\left(u_{\infty}\right)}=\int_{\mathbb{R}^{d}} f g u_{\infty} \mathrm{d} x .
$$

The kernel of $\mathcal{L}$ consists of constants and

$$
(\mathcal{L} f, f)_{L^{2}\left(u_{\infty}\right)}=-\int_{\mathbb{R}^{d}}|\nabla f|^{2} u_{\infty} \mathrm{d} x,
$$

for all sufficiently smooth functions $f$. The spectrum of $-\mathcal{L}$ is discrete consisting of eigenvalues

$$
0=\lambda_{0}<\lambda_{1}<\lambda_{2}<\ldots,
$$

and an orthonormal basis of $L^{2}\left(u_{\infty}\right)$ can be built of corresponding (normalized) eigenfunctions $\left\{\varphi_{k}\right\}_{k=0}^{\infty}$ with $\varphi_{0} \equiv 1$, see [19, Chapter 4]. For example, in the case $V(x)=\frac{1}{2}|x|^{2}, \mathcal{L}$ becomes the well known Ornstein-Uhlenbeck operator

$$
\mathcal{L} f(x)=\Delta f(x)-x \cdot \nabla f(x),
$$

the spectrum of $-\mathcal{L}$ coincides with $\mathbb{N}_{0}$, and there is an orthonormal basis of $L^{2}\left(u_{\infty}\right)$ consisting of $d$-dimensional normalized Hermite polynomials which are eigenfunctions of $-\mathcal{L}$, see $[14$, Chapter 9].

Let us first consider the case of arbitrary initial values $u_{0}$ with finite entropy. Then we may choose as initial value for $u$

$$
u_{0}(x)=\left(1+\varphi_{1}(x)\right) u_{\infty}(x),
$$


since

$$
H\left(u_{0}\right)=\int_{\mathbb{R}^{d}} \varphi_{1}^{2} u_{\infty} \mathrm{d} x<\infty,
$$

due to $\varphi_{1} \in L^{2}\left(u_{\infty}\right)$. We then have $v_{0}=1+\varphi_{1}$, and the corresponding solution $v$ of the backward Kolmogorov equation (58) is given by

$$
v(t, x)=s_{\lambda_{1}}(t) \varphi_{1}(x)+1,
$$

since

$$
\begin{aligned}
\partial_{t}\left(k *\left[v-v_{0}\right]\right)(t, x) & =\varphi_{1}(x) \partial_{t}\left(k *\left[s_{\lambda_{1}}-1\right]\right)(t, x) \\
& =-\varphi_{1}(x) \lambda_{1} s_{\lambda_{1}}(t) \\
& =\mathcal{L} \varphi_{1}(x) s_{\lambda_{1}}(t)=\mathcal{L} v(t, x) .
\end{aligned}
$$

For this solution, there holds

$$
\|v(t)-1\|_{L^{2}\left(u_{\infty}\right)}=s_{\lambda_{1}}(t)\left\|v_{0}-1\right\|_{L^{2}\left(u_{\infty}\right)}, \quad t \geq 0,
$$

that is we have equality in (56).

In the above example, the initial value $u_{0}$ and hence the solution $u$ of (1) can assume negative values as can be seen e.g. in the case of the one-dimensional Ornstein-Uhlenbeck operator (see [19, Section 4.4]).

However, we may easily modify the example presented above to cover also the case of positive solutions. Indeed, take a probability density $u_{0}$ such that $H\left(u_{0}\right)<\infty$. Then $v_{0}$ is an element of $L^{2}\left(u_{\infty}\right)$ and can be expanded as

$$
v_{0}(x)=1+\sum_{k=1}^{\infty} c_{k} \varphi_{k}(x)
$$

Then the solution for the backward Kolmogorov equation (58) is given by the series

$$
v(t, x)=1+\sum_{k=1}^{\infty} c_{k} s_{\lambda_{k}}(t) \varphi_{k}(x) .
$$

If $u_{0}$ is chosen such that $c_{1} \neq 0$, then by Parseval's identity

$$
\|v(t)-1\|_{L^{2}\left(u_{\infty}\right)}^{2}=\sum_{k=1}^{\infty} c_{k}^{2}\left(s_{\lambda_{k}}(t)\right)^{2} \geq c_{1}^{2}\left(s_{\lambda_{1}}(t)\right)^{2},
$$

which shows that in general the entropy cannot decay faster than $\left(s_{\lambda_{1}}(t)\right)^{2}$ (up to some positive constant).

In the case of the Ornstein-Uhlenbeck operator (59) in $\mathbb{R}^{d}$ the potential $V(x)=\frac{1}{2}|x|^{2}$ satisfies $\nabla^{2} V(x)=I d$ and thus the Bakry-Emery condition (12) holds with $\lambda=1$. Hence $\lambda=\lambda_{1}=1$.

We finally point out that it is an open question whether the obtained decay rates in Theorem 1.1 are optimal if $\phi \neq \phi_{2}$. 


\section{Time-discrete Fokker-Planck equation}

The purpose of this section is to show that our method also applies to the time-discretized Fokker-Planck equation

$$
\frac{1}{\tau}\left(u\left(t_{n}, x\right)-u\left(t_{n-1}, x\right)\right)-\left(\mathcal{L}^{*} u\right)\left(t_{n}, x\right)=0, \quad n \in \mathbb{N}, x \in \mathbb{R}^{d},
$$

with initial condition

$$
u(0, x)=u_{0}(x), \quad x \in \mathbb{R}^{d} .
$$

Here we use the backward difference scheme for the usual time derivative $\partial_{t}$ with time step $\tau$, that is $t_{n}=n \tau, n \in \mathbb{N}_{0}$. The Fokker-Planck operator $\mathcal{L}^{*}$ is as before, see (36) for its definition.

By means of the crucial estimate from Remark 3.1 for the generating function $\phi_{\beta}$ and the associated entropy we are able to improve, in the case of a power type entropy, the rate of (exponential) convergence known in the literature for general entropies. So also in the timediscrete case the decay rates become better with higher integrability of the initial datum.

We will prove the following theorem.

Theorem 6.1 Suppose that $V \in C^{2}\left(\mathbb{R}^{d}\right)$ satisfies condition (12) for some $\lambda>0$ and is such that $e^{-V} \in L^{1}\left(\mathbb{R}^{d}\right)$. Let $u_{0} \in L^{1}\left(\mathbb{R}^{d}\right)$ be a probability density and $u_{\infty}$ be defined as in (8). Let further $\tau>0$ and $t_{n}=n \tau, n \in \mathbb{N}_{0}$. Let $\beta \in(1,2]$ and $\phi_{\beta}$ be defined as in (11). Let $H(u)$ be the corresponding relative entropy associated with $u_{\infty}$. Assume that $H\left(u_{0}\right)<\infty$ and that the positive solution $u$ of (61), (62) is sufficiently smooth. Then

$$
H\left(u\left(t_{n}\right)\right) \leq\left(\frac{1}{1+\frac{2 \tau \lambda}{\beta}}\right)^{\beta n} H\left(u_{0}\right), \quad n \in \mathbb{N} .
$$

Moreover,

$$
\left\|u\left(t_{n}\right)-u_{\infty}\right\|_{L^{1}\left(\mathbb{R}^{d}\right)} \leq \sqrt{\frac{2}{\beta(\beta-1)}}\left(\frac{1}{1+\frac{2 \tau \lambda}{\beta}}\right)^{\frac{\beta n}{2}} \sqrt{H\left(u_{0}\right)}, \quad n \in \mathbb{N} .
$$

To compare this result with what is known in the literature, we remark that if one replaces $\phi_{\beta}$ by an arbitrary admissible generating function $\phi$, then it is already known that

$$
H\left(u\left(t_{n}\right)\right) \leq\left(\frac{1}{1+2 \tau \lambda}\right)^{n} H\left(u_{0}\right), \quad n \in \mathbb{N},
$$

see [9, Remark 5.9]; we also refer to [2]. Observe that for $\beta \in(1,2]$

$$
\left(\frac{1}{1+\frac{2 \tau \lambda}{\beta}}\right)^{\beta}<\frac{1}{1+2 \tau \lambda}
$$

In fact, setting $\delta=\frac{1}{\beta}$ the function $g(y)=y^{\delta}$ is strictly concave in $[0, \infty)$ and thus we have for $a>0$

$$
(1+a)^{\delta}<1^{\delta}+\delta 1^{\delta-1} a=1+\frac{a}{\beta} .
$$

The claim (66) then follows easily from this inequality with $a=2 \tau \lambda$. 


\subsection{The fundamental identity for the discrete time derivative}

For fixed $\tau>0$ we define the operator $D$ by

$$
D u(t)=\frac{1}{\tau}(u(t)-u(t-\tau))
$$

Let $I \subset \mathbb{R}$ be an interval, $\psi \in C^{1}(I), N \in \mathbb{N}, J:=\{n \tau: n=0, \ldots, N\}$, and $u: J \rightarrow I$. Then there holds an analogue of the fundamental identity (27), more precisely, we have for all $t \in J \backslash\{0\}$

$$
\psi^{\prime}(u(t))(D u)(t)=D(\psi(u))(t)+\frac{1}{\tau}\left(\psi(u(t-\tau))-\psi(u(t))-\psi^{\prime}(u(t))[u(t-\tau)-u(t)]\right) .
$$

This is folklore and follows directly from the definition of $D$. We remark that corresponding identities also hold for operators which are discrete in space, e.g. the Laplacian on graphs ([4]).

As a direct consequence of (67) we obtain for convex functions $\psi$ that

$$
\psi^{\prime}(u(t))(D u)(t) \geq D(\psi(u))(t)
$$

\subsection{Proof of Theorem 6.1}

Let $u$ be the solution of problem (61), (62). We set again $v=u / u_{\infty}$ and $v_{0}=u_{0} / u_{\infty}$. Multiplying $(61)$ by $\phi^{\prime}\left(v\left(t_{n}\right)\right)$, integrating over $\mathbb{R}^{d}$ and integrating by parts we obtain (cf. the beginning of Section 3)

$$
\tau^{-1} \int_{\mathbb{R}^{d}}\left(v\left(t_{n}\right)-v\left(t_{n-1}\right)\right) \phi^{\prime}\left(v\left(t_{n}\right)\right) u_{\infty} \mathrm{d} x=-\int_{\mathbb{R}^{d}} \phi^{\prime \prime}\left(v\left(t_{n}\right)\right)\left|\nabla v\left(t_{n}\right)\right|^{2} u_{\infty} \mathrm{d} x
$$

Let us first recall how the general estimate (65) can be derived. Since $\phi$ is convex, we can apply the convexity inequality (68) for the discrete time derivative to estimate the left-hand side of (69) as follows (see also [9, Section 5.3]):

$$
\begin{aligned}
\int_{\mathbb{R}^{d}}\left(v\left(t_{n}\right)-v\left(t_{n-1}\right)\right) \phi^{\prime}\left(v\left(t_{n}\right)\right) u_{\infty} \mathrm{d} x & \geq \int_{\mathbb{R}^{d}}\left(\phi\left(v\left(t_{n}\right)\right)-\phi\left(v\left(t_{n-1}\right)\right)\right) u_{\infty} \mathrm{d} x \\
& =H\left(u\left(t_{n}\right)\right)-H\left(u\left(t_{n-1}\right)\right) .
\end{aligned}
$$

As in the continuous case, the right-hand side of (69) can be estimated from above by means of the convex Sobolev inequality, Theorem 2.2. This yields

$$
\tau^{-1}\left(H\left(u\left(t_{n}\right)\right)-H\left(u\left(t_{n-1}\right)\right)\right) \leq-2 \lambda H\left(u\left(t_{n}\right)\right)
$$

which shows that $H(u)$ is nonincreasing and which is equivalent to

$$
H\left(u\left(t_{n}\right)\right) \leq \frac{1}{1+2 \tau \lambda} H\left(u\left(t_{n-1}\right)\right), \quad n \in \mathbb{N} .
$$

This inequality in turn implies the general estimate (65).

In order to obtain the better estimate for $\phi=\phi_{\beta}$ we proceed analogously to Subsection 3.2. The key idea is again to use the fundamental identity (now in the form (67)) in its full strength. 
Denoting again the discrete time derivative by $D$, by (67) we can write the left-hand side of (69) in the form

$$
\begin{aligned}
\int_{\mathbb{R}^{d}}(D v)\left(t_{n}\right) & \phi^{\prime}\left(v\left(t_{n}\right)\right) u_{\infty} \mathrm{d} x=\int_{\mathbb{R}^{d}} D(\phi(v))\left(t_{n}\right) u_{\infty} \mathrm{d} x \\
& +\tau^{-1} \int_{\mathbb{R}^{d}}\left(\phi\left(v\left(t_{n-1}\right)\right)-\phi\left(v\left(t_{n}\right)\right)-\phi^{\prime}\left(v\left(t_{n}\right)\right)\left[v\left(t_{n-1}\right)-v\left(t_{n}\right)\right]\right) u_{\infty} \mathrm{d} x \\
= & : A_{1}(n)+A_{2}(n) .
\end{aligned}
$$

Evidently,

$$
A_{1}(n)=D(H(u))\left(t_{n}\right) .
$$

The structure of the second term in (71) is the same as that of the third term on the right-hand side of (42). So we can follow the line of arguments given in Subsection 3.2. We have

$$
\begin{aligned}
A_{2}(n)= & \tau^{-1}\left(H\left(u\left(t_{n-1}\right)\right)-H\left(u\left(t_{n}\right)\right)\right)-\frac{\beta}{\tau} \int_{\mathbb{R}^{d}}\left(v\left(t_{n}\right)^{\beta-1}-1\right)\left[v\left(t_{n-1}\right)-v\left(t_{n}\right)\right] u_{\infty} \mathrm{d} x \\
= & \tau^{-1}\left(H\left(u\left(t_{n-1}\right)\right)-H\left(u\left(t_{n}\right)\right)\right)+\frac{\beta}{\tau} H\left(u\left(t_{n}\right)\right) \\
& -\frac{\beta}{\tau} \int_{\mathbb{R}^{d}}\left(v\left(t_{n}\right)^{\beta-1} v\left(t_{n-1}\right)-v\left(t_{n}\right)\right) u_{\infty} \mathrm{d} x \\
\geq & \tau^{-1}\left(H\left(u\left(t_{n-1}\right)\right)+(\beta-1) H\left(u\left(t_{n}\right)\right)\right)-\frac{\beta}{\tau} H\left(u\left(t_{n}\right)\right)^{\frac{\beta-1}{\beta}} H\left(u\left(t_{n-1}\right)\right)^{\frac{1}{\beta}},
\end{aligned}
$$

where we used the inequality from Remark 3.1. Combining this, (72) and (71) and setting $w(t)=H(u(t))^{\frac{1}{\beta}}$ we see that

$$
\begin{aligned}
\int_{\mathbb{R}^{d}}(D v)\left(t_{n}\right) \phi^{\prime}\left(v\left(t_{n}\right)\right) u_{\infty} \mathrm{d} x \geq D(H(u))\left(t_{n}\right) \\
\quad+\tau^{-1}\left(H\left(u\left(t_{n-1}\right)\right)+(\beta-1) H\left(u\left(t_{n}\right)\right)\right)-\frac{\beta}{\tau} H\left(u\left(t_{n}\right)\right)^{\frac{\beta-1}{\beta}} H\left(u\left(t_{n-1}\right)\right)^{\frac{1}{\beta}} \\
=D\left(w^{\beta}\right)\left(t_{n}\right)+\tau^{-1}\left(w\left(t_{n-1}\right)^{\beta}-w\left(t_{n}\right)^{\beta}-\beta w\left(t_{n}\right)^{\beta-1}\left[w\left(t_{n-1}\right)-w\left(t_{n}\right)\right]\right) \\
=\beta w\left(t_{n}\right)^{\beta-1}(D w)\left(t_{n}\right),
\end{aligned}
$$

where in the last step we applied the fundamental identity (67).

Altogether, we obtain

$$
\beta w\left(t_{n}\right)^{\beta-1}(D w)\left(t_{n}\right) \leq-2 \lambda w\left(t_{n}\right)^{\beta}, \quad n \in \mathbb{N} .
$$

The sequence $\left(H\left(u\left(t_{n}\right)\right)\right)_{n \in \mathbb{N}_{0}}$ is nonnegative and nonincreasing, so $\left(w\left(t_{n}\right)\right)_{n \in \mathbb{N}_{0}}$ enjoys the same properties. Consequently, if $w\left(t_{n_{*}}\right)=0$ for some $n_{*} \in \mathbb{N}_{0}$ then $w\left(t_{n}\right)=0$ for all $n \geq n_{*}$ and thus the asserted entropy estimate trivially holds for all $n \geq n_{*}$. It remains to look at those times $t_{n}$, where $w\left(t_{n}\right)>0$.

So assume that $w\left(t_{n}\right)>0$ for all $n \in\{0, \ldots, N\}$. Then (73) implies that

$$
\tau^{-1}\left(w\left(t_{n}\right)-w\left(t_{n-1}\right)\right) \leq-\frac{2 \lambda}{\beta} w\left(t_{n}\right), \quad n \in\{1, \ldots, N\},
$$


and thus

$$
H\left(u\left(t_{n}\right)\right) \leq\left(\frac{1}{1+\frac{2 \tau \lambda}{\beta}}\right)^{\beta n} H\left(u_{0}\right), \quad n \in\{1, \ldots, N\} .
$$

Since $H\left(u\left(t_{n}\right)\right)=0$ if and only $w\left(t_{n}\right)=0$ and by the remarks following (73), the last inequality even holds for all $n \in \mathbb{N}$, thus proving (63). The second assertion of the theorem follows from (63) and the Csiszár-Kullback-Pinsker inequality stated in Theorem 2.1.

\section{References}

[1] Arnold, A.; Markowich, P.; Toscani, G.; Unterreiter, A.: On convex Sobolev inequalities and the rate of convergence to equilibrium for Fokker-Planck type equations. Comm. Partial Differential Equations 26 (2001), 43-100.

[2] Arnold, A.; Unterreiter, A.: Entropy decay of discretized Fokker-Planck equations. I. Temporal semidiscretization. Comput. Math. Appl. 46 (2003), 1683-1690.

[3] Clément, Ph.; Nohel, J.A.: Asymptotic behavior of solutions of nonlinear Volterra equations with completely positive kernels. SIAM J. Math. Anal. 12 (1981), 514-534.

[4] Dier, D.; Kassmann, M.; Zacher, R.: Discrete versions of the Li-Yau gradient estimate. Preprint 2017. Available online at https://arxiv.org/abs/1701.04807.

[5] Feller, W.: An introduction to probability theory and its applications. Vol. II. Second edition. John Wiley \& Sons, Inc., New York, 1971.

[6] Gripenberg, G.; Londen, S.-O.; Staffans, O.: Volterra integral and functional equations. Encyclopedia of Mathematics and its Applications, 34. Cambridge University Press, Cambridge, 1990.

[7] Hahn, M.; Kobayashi, K.; Umarov, S.: SDEs driven by a time-changed Lévy process and their associated time-fractional order pseudodifferential equations. J. Theoret. Probab. 25 (2012), 262-279.

[8] Jordan, R.; Kinderlehrer, D.; Otto, F.: The variational formulation of the Fokker-Planck equation. SIAM J. Math. Anal. 29 (1998), 1-17.

[9] Jüngel, A.: Entropy methods for diffusive partial differential equations. Springer, 2016.

[10] Kemppainen, J.; Siljander, J.; Vergara, V.; Zacher, R.: Decay estimates for time-fractional and other nonlocal in time subdiffusion equations in $\mathbb{R}^{d}$. Math. Ann. 366 (2016), 941-979.

[11] Kemppainen, J.; Siljander, J.; Zacher, R.: Representation of solutions and large-time behavior for fully nonlocal diffusion equations. J. Differential Equations 263 (2017), 149-201.

[12] Kilbas, A. A.; Srivastava, H. M.; Trujillo, J. J.: Theory and applications of fractional differential equations. Elsevier, 2006.

[13] Kochubei, A. N.: Distributed order calculus and equations of ultraslow diffusion. J. Math. Anal. Appl. 340 (2008), 252-281.

[14] Lorenzi, L.; Bertoldi, M.: Analytical Methods for Markov semigroups. Pure and Applied Mathematics (Boca Raton), 283. Chapman \& Hall/CRC, Boca Raton, FL, 2007.

[15] Markowich, P.A.; Villani, C.: On the trend to equilibrium for the Fokker-Planck equation: an interplay between physics and functional analysis. VI Workshop on Partial Differential Equations, Part II (Rio de Janeiro, 1999). Mat. Contemp. 19 (2000), 1-29.

[16] Meerschaert, M.M.; Nane, E.; Vellaisamy, P.: Fractional Cauchy problems on bounded domains. Ann. Probab. 37 (2009), 979-1007. 
[17] Metzler, R.; Klafter, J.: The random walk's guide to anomalous diffusion: a fractional dynamics approach. Phys. Rep. 339 (2000), 1-77.

[18] Metzler, R.; Klafter, J.: The restaurant at the end of the random walk: recent developments in the description of anomalous transport by fractional dynamics. J. Phys. A 37 (2004), R161-R208.

[19] Pavliotis, G. A.: Stochastic processes and applications. Diffusion processes, the Fokker-Planck and Langevin equations. Texts in Applied Mathematics, 60. Springer, 2014.

[20] Prüss, J.: Evolutionary Integral Equations and Applications. Monographs in Mathematics 87, Birkhäuser, Basel, 1993.

[21] Toscani, G.: Entropy production and the rate of convergence to equilibrium for the Fokker-Planck equation. Quart. Appl. Math. 57 (1999), 521-541.

[22] Vergara, V.; Zacher, R.: Optimal decay estimates for time-fractional and other non-local subdiffusion equations via energy methods. SIAM J. Math. Anal. 47 (2015), 210-239.

[23] Zacher, R.: Boundedness of weak solutions to evolutionary partial integro-differential equations with discontinuous coefficients. J. Math. Anal. Appl. 348 (2008), 137-149.

[24] Zacher, R.: A De Giorgi-Nash type theorem for time fractional diffusion equations. Math. Ann. 356 (2013), 99-146.

[25] Zacher, R.: Maximal regularity of type $L_{p}$ for abstract parabolic Volterra equations. J. Evol. Equ. 5 (2005), $79-103$.

[26] Zacher, R.: Weak solutions of abstract evolutionary integro-differential equations in Hilbert spaces. Funkcialaj Ekvacioj 52 (2009), 1-18.

Jukka Kemppainen, Applied and Computational Mathematics, Pentti Kaiteran katu 1, PO Box 8000, FI-90014 University of Oulu, Finland, e-mail: Jukka.T.Kemppainen@oulu.fi

Rico Zacher, Ulm University, Institute of Applied Analysis, 89069 Ulm, Germany, e-mail: rico.zacher@uniulm.de 